\title{
INTELLIGENT HOME MONITORING USING RSSI IN WIRELESS SENSOR NETWORKS
}

\author{
FIRDOUS KAUSAR, EISA AL EISA, IMAM BAKHSH \\ Department of Computer Science, \\ College of Computer and Information Sciences, \\ Imam University, \\ Riyadh, Saudi Arabia. \\ firdous.kausareccis.imamu.edu.sa, aleisa@ccis.imamu.edu.sa and \\ ibakhsh@ccis.imamu.edu.sa
}

\begin{abstract}
Automated systems have become deeply involved in our daily life these days. Every day new researches and solutions are introduced in the worldwide to utilize computer systems to satisfy human needs. In this project we propose a "Intelligent Home Security Monitoring System" (IHMS) using RSSI in Wireless Sensor Networks. This paper presents a system using Wireless sensor networks (WSNs) enabled smart home environments to create pervasive and ubiquitous applications, which give scalable services and context-aware to the end users. This system develops an application and reports its implementation on real WSN to provide remote home security. There is a plan to use variations in received signal strength indicator (RSSI) value to find intrusion activity at home. Architecture of an application has been presented consisting of Moteivs Tmote Sky motes and a base station. The application sends a piece of alarm as a short message (SMS) on cellular phone of the homeowner in case of any intrusion activity has been detected at home.
\end{abstract}

\section{KEYWORDS}

Home Security System, Wireless Sensor Network, Received Signal Strength Indicator, Remote Monitoring

\section{INTRODUCTION}

Smart home environments are the integration of technology and services through home networking for automating, improving, security, safety, communication, comfort and energy saving. A smart home based on WSN is using solid deployment of sensors to provide insinuate and precise monitoring.

In these days, home security has been become an important issue due to high rate of crimes and everybody has intended to acquire reasonable measures to prevent intrusion. Therefore the motivation of this project is to investigate a cost effective solution that provides home monitoring and enable home security against intrusion in the absence of the home owner.

In addition, building monitoring systems exist in most of the commercial buildings with the first duty of providing convenience and safety. However, implementation and maintenance of wired systems are time consuming, error-prone and costly (e.g., for existing construction: $2.2 \$$ per linear foot and for new construction: $0.67 \$$ per linear foot).

Another issue is when some crisis happens in a building, such as earth-quack or fire. Since the damage to the construction make the backbone system fail, the whole system stops operating. The new technology of wireless sensor network has brought a new level of building monitoring systems by saving the cost and time of implementation and maintenance, providing more safety DOI : $10.5121 /$ ijenc. 2012.4603 
International Journal of Computer Networks \& Communications (IJCNC) Vol.4, No.6, November 2012 and robustness and making these systems more stable in "hazardous" circumstance is due to the nature of WSN. In WSN each node in the network is independent of the other nodes, they are battery powered, small in size with attached sensors [1].

The RSSI relatively receives signal strength in a wireless environment. There is a direct link between the radio frequency (RF) power level received by one node and the RSSI indication [2]. The RSSI is a relative measurement of the strength of a radio signal's energy as measured by WSNs and it is generally hidden to the device's user containing a receiver due generic radio receiver technology metric, but it is well-known to users of wireless networking of IEEE 802.11 protocol family.

In this paper we develop a web-based application using WSN to provide the remote monitoring of home security. We implement the application in a real WSN environment using moteiv tmotes. It can detect the theft, and in case of any dangerous condition has been detected, it can send warning SMS on cellular phone of the user.

\section{Literature Review}

Subhas C. Mukhopadhyay et al. [3] presents review of several wireless sensors, which are used for home monitoring particularly to look after aged people. The monitoring system is established on the combination of several sensors, and it has the ability of broadcasting data via wireless communication. The central processor collects data and stores all data for current requirement and for future purpose. The system stores the habit of life style of a person. The system compares collected data with stored pattern, which depends on situations and actions are already defined like abnormal or unusual. In case of any abnormal activity, the system detects it and generates an alarming or warning or SMS and it is transmitted to the caregiver. This wireless sensing system is available for this type of application with low cost and has great potential to save human lives of old age people.

Wei-Chung Teng et al. [4] proposed the design and implementation of a residential gateway: MyServer, which gives home security services. The system driven by peripherals connected through WSNs. MyServer structural designed to operate on Message Oriented Middleware (MOM) with six clearly defined core service modules.

Sukun Kim et al. [5] present an active research in a WSN for Structural Health Monitoring (SHM). Requirements are identified to acquire data for sufficient quality to have a real scientific value to the researchers for structural health monitoring. The collected data matches with theoretical models and as well as with previous studies of the bridge. The deployment is the biggest WSN for SHM.

Alan Mainwaring et al. [6], provides a depth study regarding using of WSNs to real environment habitat monitoring. The developed system covers the hardware design of nodes and sensor network for remote access and its management. Architecture of system is suggested to deal with the requirements of habitat monitoring. The example of system architecture for monitoring of sea-bird nesting environment and activities is presented. As per presently installment, the network has 32 notes on a small island off the coast of Maine useful live data on web. The application driven design serves to identify important areas of additional work in communications, network re-tasking, data sampling, and health monitoring.

Huiping Huang et al. [7], presents a remote home security alarm system with a solution for set-up low power consumption. The system detects the theft, fir, and leakage of raw-gas by using the WSN and GSM technology. In case of any abnormality, the system sends alarm message remotely. The single chip C5081F310 is hardware of the system, which communicates via wireless using chip CC1100 with SIMENS TC35 GSM module. The software of the system developed using C51 language, which has capability of gathering, receiving and sending data via wireless. In case of detection of some dangerous condition, it sends alarming SMS to users of 
International Journal of Computer Networks \& Communications (IJCNC) Vol.4, No.6, November 2012

cellular phone. With easy-usage advantages, low-power consumption, reliability, and complement wireless, this system can be used for practical value in other fields too.

Jianjun Chen et al. [8], by using o_-the-shelf WLAN components, those are commercially available, described implementation of indoor surveillance system. This security system constantly scanned the environment. It had the ability to deliver real-time alarm signals on the basis of detected changes in the received signal strength values. The experimental results showed promising intrusion detection capabilities but the exact performance limitation and strength of this surveillance system is yet to be investigated.

Youssouf Zatout et al. [9] present solution of saving the energy of wireless sensor in a mixed environment for Home Monitoring. It suggests a design and an implementation of three-tier sensor network solution, which uses energy efficiently for home applications. The network consists of heterogeneous sensors e.g. environmental, medical, and video/audio sensors. The base solution is to organize the sensors into different groups as per their particular functions and roles. According to intelligent behavior of the sensor, the activity duration and communication are reduced at the same time.

Dong-Sun Kim et al. [10] describes a time synchronized forwarding protocol (TSFP), which is used for remote control home devices. For transferring data to another node, WSN has an extremely large latency, so, it uses TSFP. TSFP provides scalability by a self-organization function based on a virtual sensor line and mainly it utilizes distributed time division multiple access (TDMA). On the large scale WSNs, it reduces transmission latency and energy consumption.

A. Gaddam et al. [11] presents smart digital home monitoring system by using a bed sensor integrated with a wireless device. The Based system that uses wireless sensors to monitor electrical appliances, for example is it closed or open. To make the system exible by adding sensors of different types Using selective activity monitoring (SAM) system.

Yanjun Li. [12] described a blueprint of a novel reactive WSN for monitoring wild-fire and evaluated robustness, reactivity, longevity, and reliability of the network. The contribution of the paper is to design a sensor network that can meet the goal of reliability, reactivity, and that proves acceptable robustness and relatively longer lifetime network life.

Yu-Tso Chen et al. [13] proposed a Closer Tracking Algorithm (CTA) to pinpoint user of mobile inside the home by inquiring regarding RSSI solutions for indoor localization. The investigation results depict that projected Closer Tracking Algorithm has the ability to reveal the accurate position with error 6 distances less than 1 meter. If the distance is less than one member, the suggested Closer Tracking Algorithm has minimum $85 \%$ precision at the same time. The proposed Closer Tracking Algorithm was implemented by using ZigBee CC2431 modules.

\section{Proposed System Design}

This section presents the design of our proposed Intelligent Home Monitoring System using RSSI in Wireless Sensors Networks (IHMS). We develop a IHMS to detect potential disastrous problems, sending SMS and email to distant homeowner in order to notify the likelihood of robbery at home.

Following are the features of IHMS:

- Generate real time notification through SMS and Email for any kind of intrusion at home.

- Try to minimize the rate of false positives and false negatives.

- Draw a real-time dynamic graph represent RSSI average values. 
The operating environment of IHMS consists of both software and hardware components.

Software components include TinyOS and Server on the internet to send SMS and Email when the system detects any threats. Hardware components include Tmote as sensor nodes and a laptop or PC with internet connection.

Figure 3.1 presents the use case diagram of IHSMS. There are four actors in IHMS and they are presented as the following:

- Homeowner: It is the person who is responsible for controlling the main activities of the system (Enable system and Disable system).

- Server: It is the web application that sends SMS and Email to the Homeowner.

- Base Station: It is the PC or laptop that receives data packets from Sensor Motes. Then calculate RSSI value of received packets, and store RSSI values in Data Base, Monitor variation of RSSI.

- Sensor Motes: It is the device that will sense the around environment then based on the result of sensing will send data packets to base Station.

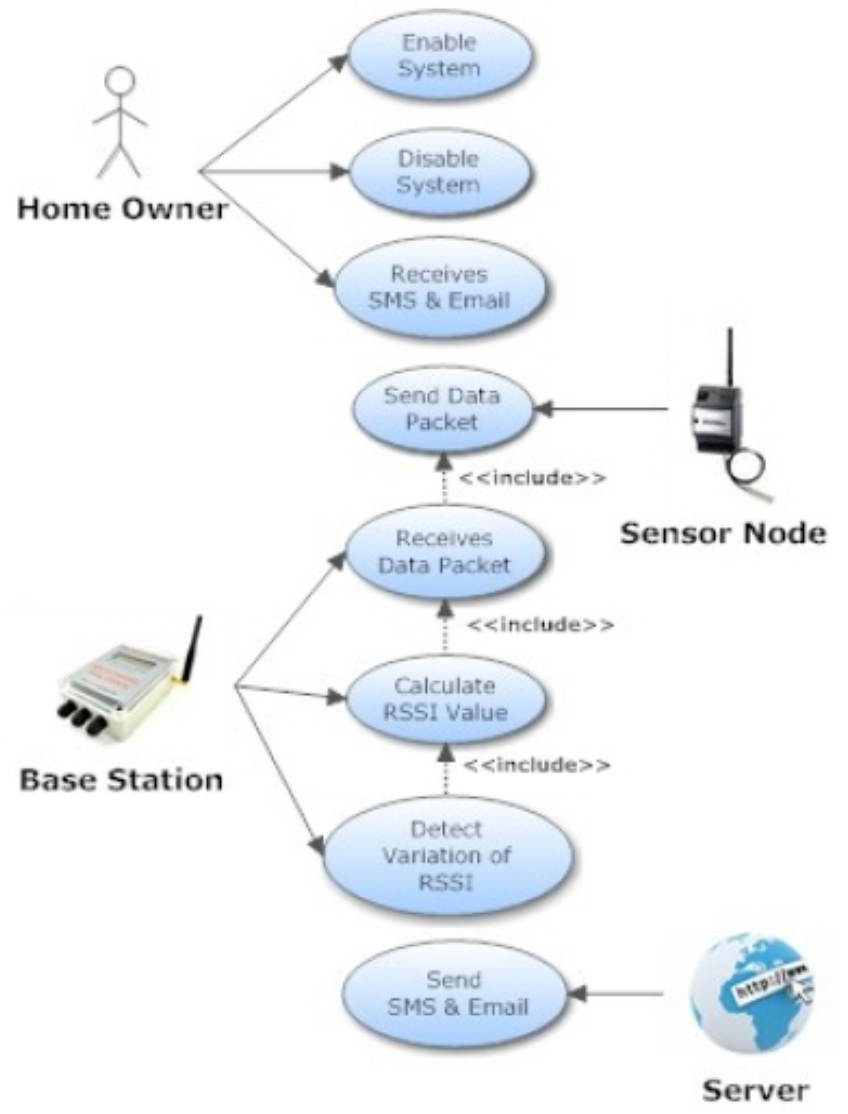

Figure 3.1: Use Case Diagram

The Enable system use case enables the homeowner to activate the system. This use case starts when Homeowner decides to enable the system. Homeowner chooses Enable operation of the user interface by clicking on the enable button. IHMS turns on and Base Station starts receiving data packets from the Sensor Motes. 
International Journal of Computer Networks \& Communications (IJCNC) Vol.4, No.6, November 2012

The Disable system use case enables the homeowner to close the system. This use case starts when Homeowner decides to disable the system. Homeowner chooses disable operation of the user interface by clicking on the disable button. The system sends a message to Sensor Motes to go to sleep mode (do not send any more data packets) and exit the application.

The Receive SMS and Email use case enable the homeowner to receive SMS and email from the server. Base Station sends warning to server. The server sends SMS and Email to Homeowner. The homeowner will receive the notification messages.

The Send Data Packet use case allows the Sensor Motes to Send Data Packet to Base Station. Homeowner Enable the system. Sensor Motes send a data packet after a specific time interval to the Base Station.

The Receive Data Packet use case provides Base-Station to Receive Data Packet from a Sensor Motes. The Calculate RSSI values Use Case enables the Base Station to calculate RSSI values from received data packet.

The Detect variation of RSSI use case enables the Base Station to detect variation in the currently received RSSI value with previously stored RSSI values in database. Base Station receives data packet from sensor motes. It calculates RSSI value of each received packet and store in its database. Then Base Station compares the currently received RSSI value with previously stored RSSI values in order to determine that variation should not cross the predefined threshold RSSI value. If RSSI values remain in the acceptable range and variation is not large from previously recorded RSSI values, then Base Station keeps receiving more data packets from sensor motes. If the RSSI value is not in acceptable range then Base Station sends warning to server. The server sends SMS and email to the homeowner for notifying the intrusion activity at home.

The basic operations of IHMS are shown in the following pseudocode.

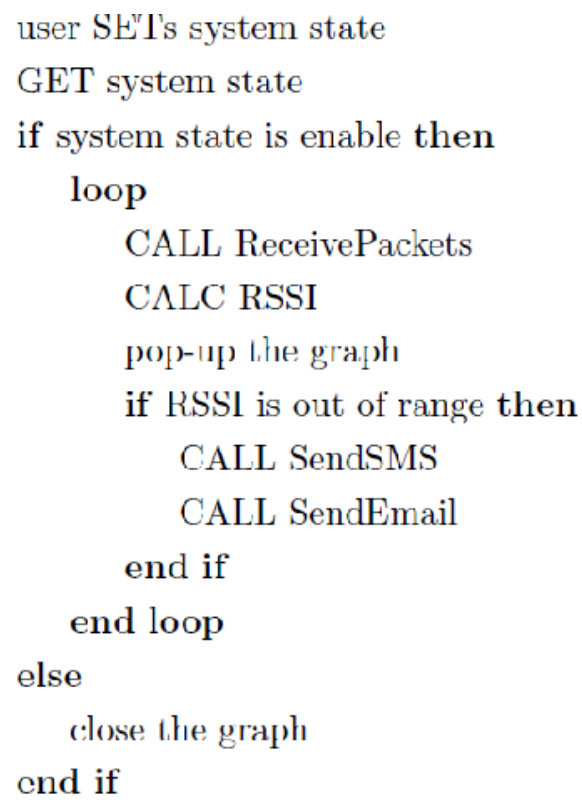


International Journal of Computer Networks \& Communications (IJCNC) Vol.4, No.6, November 2012 Figure 3.2 shows the state chare diagram of IHMS which describes the different states of a component in a system.

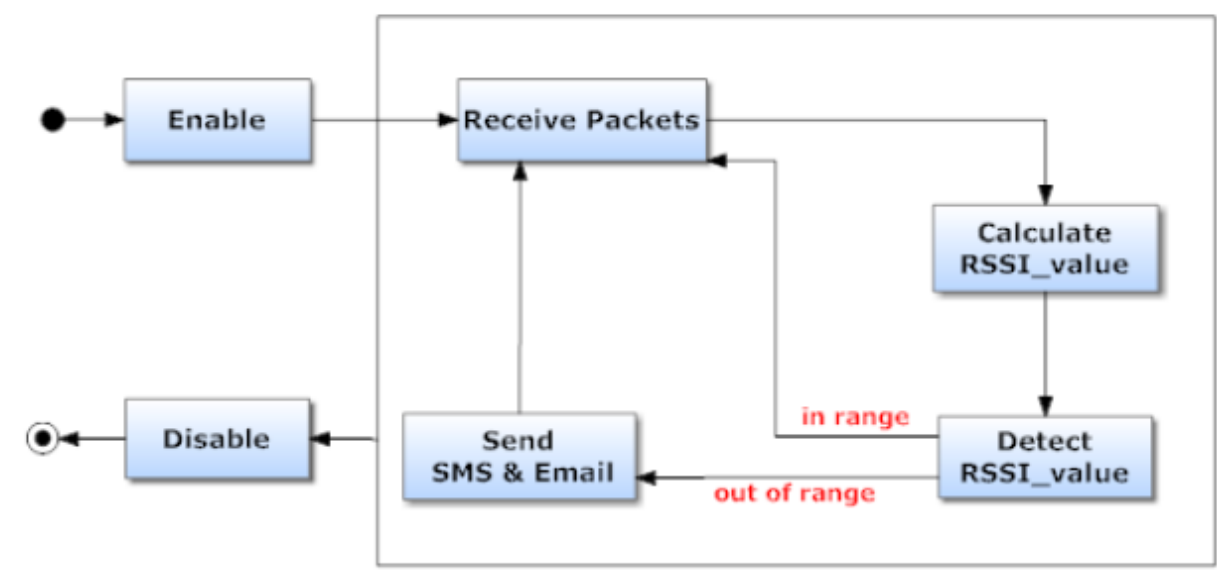

Figure 3.2: State Chart Diagram of IHMS

\section{IMPLEMENTATION}

This section presents the implementation details of our proposed IHMS. We use Java as a programming language for Web application development and NesC to program our Tmote sensor motes running TinyOS operating system.

In the interface the homeowner can login to the system by entering his password as shown in Figure 4.1. This property ensures the authentication and the security of the system. After authenticating by the system, user can choose one of operations that provide to control the system by Enable and Disable it as shown in Figure 4.2. When homeowner enables the system, he gets a dynamic graph presents real time RSSI form different sensor motes as shown in Figure 4.3. If the homeowner forgets the password, the system asks him to insert phone number and check if it is one of the stored contact information. Then, the system sends one time password to that phone number so the homeowner can set a new password as shown in Figure 4.4.

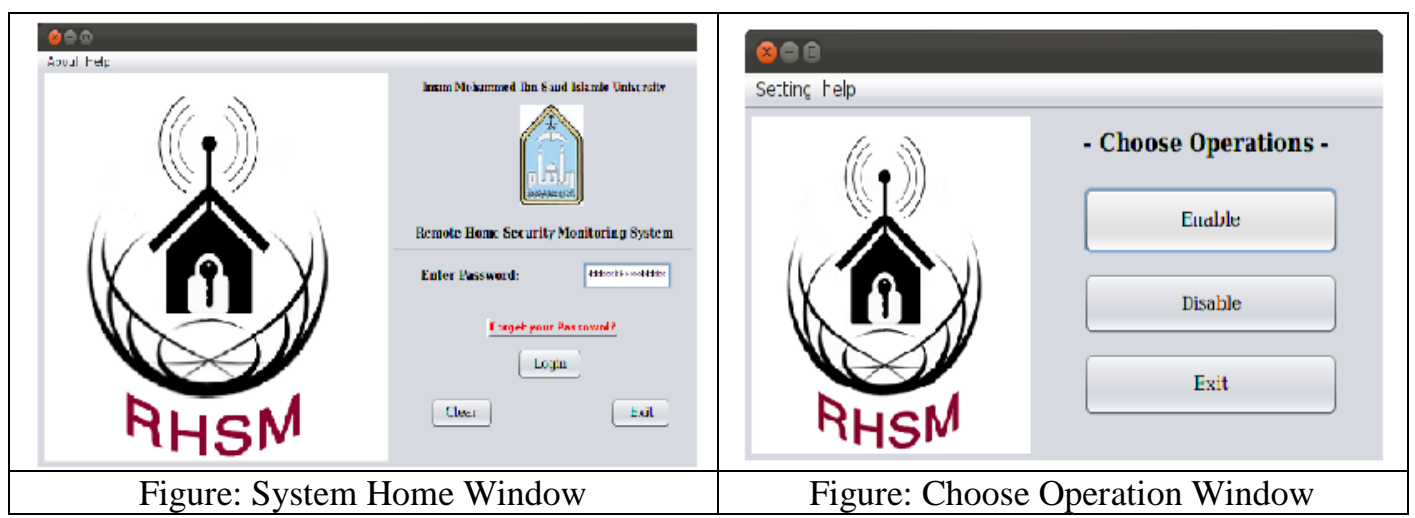




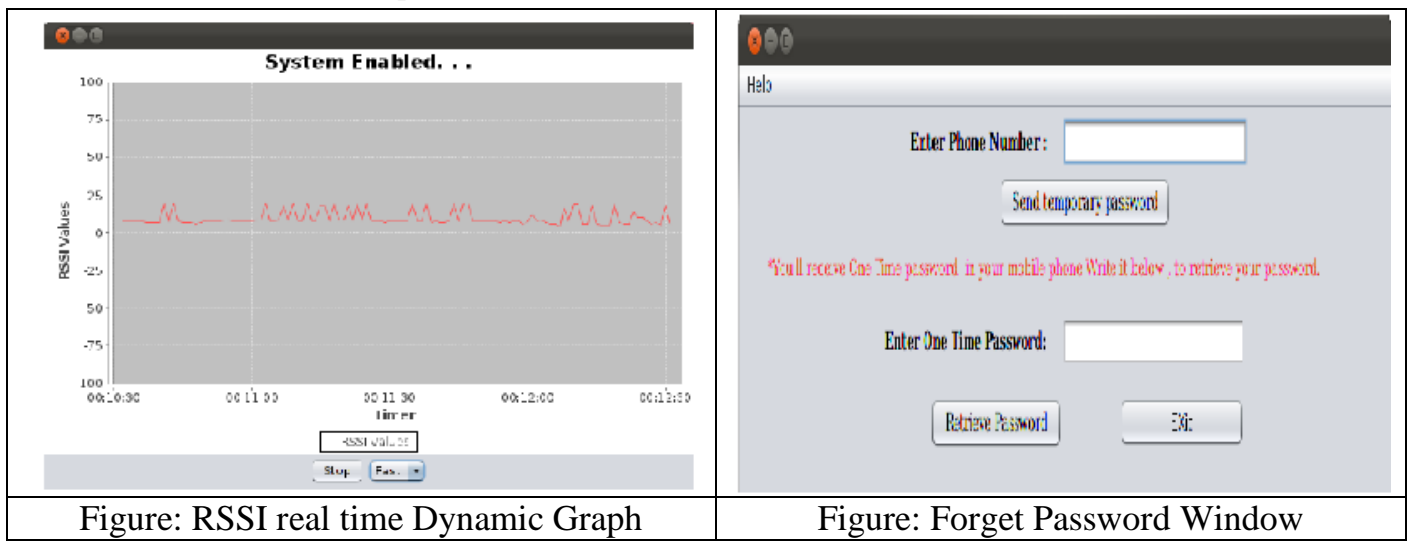

\section{EXPERIMENTAL EVALUATION}

Under this section the proposed system takes an RSSI value to find the intrusion activity at home. RSSI is variable value. So, it is based on the experiences. The experiment has been done 63 times to find the accurate range of intrusion activity at home. They have been done for three configurations setup.

\subsection{First Configuration}

In this configuration, sensors are away from the Base-Station 2.5 meters and the Base-Station rises from the ground 1 meter show in Figure 5.1.

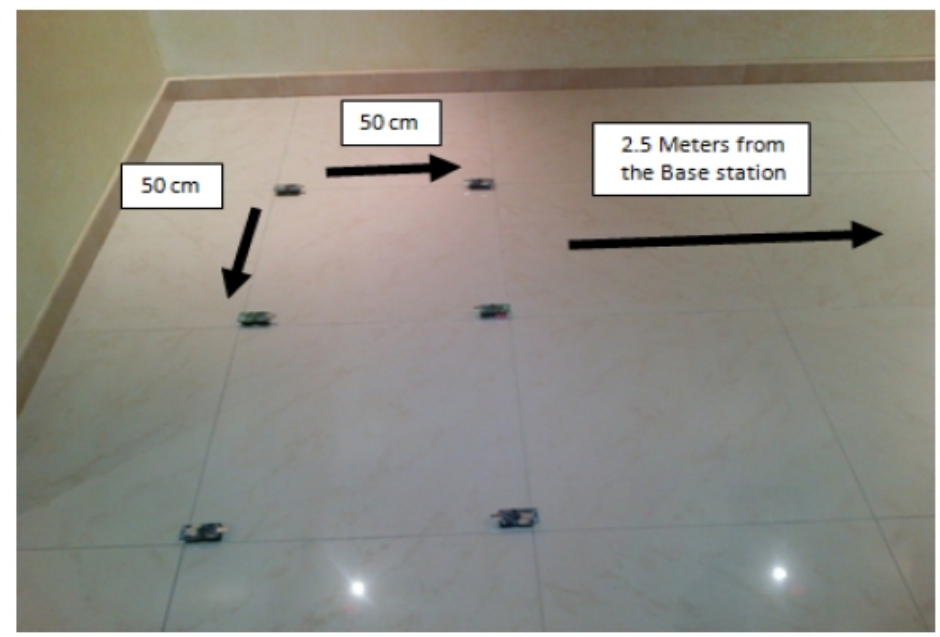

Figure 5.1: First sensors configuration

In this configuration 21 experiences have been done. The Cases are:

1. Adult (Quickly movement): 3 tests.

2. Adult (Slow movement): 3 tests.

3. Children (Quick movement): 3 tests.

4. Children (Slow movement): 3 tests.

5. Cat: 3 tests.

6. Obstacle: 3 tests. 
International Journal of Computer Networks \& Communications (IJCNC) Vol.4, No.6, November 2012 Table 5.1 illustrates the RSSI range for each experiment.

\begin{tabular}{|l||c|c|c|}
\hline Tested Object & Test 1 & Test2 & Test3 \\
\hline adult (Quickly) & -9 to -34 & -9 to -40 & -10 to -34 \\
\hline adult (Slowly) & -9 to -31 & -9 to -40 & -9 to -34 \\
\hline child (Quickly) & -16 to -44 & -16 to -35 & -12 to -33 \\
\hline child (Slowly) & -10 to -37 & -15 to -35 & -15 to -42 \\
\hline cat & -14 to -36 & -14 to -29 & -15 to -26 \\
\hline obstacle & -13 to -19 & -15 to -19 & -15 to -19 \\
\hline no movement & -9 to -18 & -9 to -17 & -9 to -17 \\
\hline
\end{tabular}

Table 5.1: First configuration testing data

As a result of several experiments, it is found that if RSSI $\leq 9$, there is a high probability of intrusion activity at home. Therefore SMS and Email are sent to homeowner if RSSI $\mathbf{1 9}$. Mean, variance and standard deviation of RSSI value for experiments is shown in Figure 5.2, Figure 5.3, Figure 5.4 and Figure 5.5.

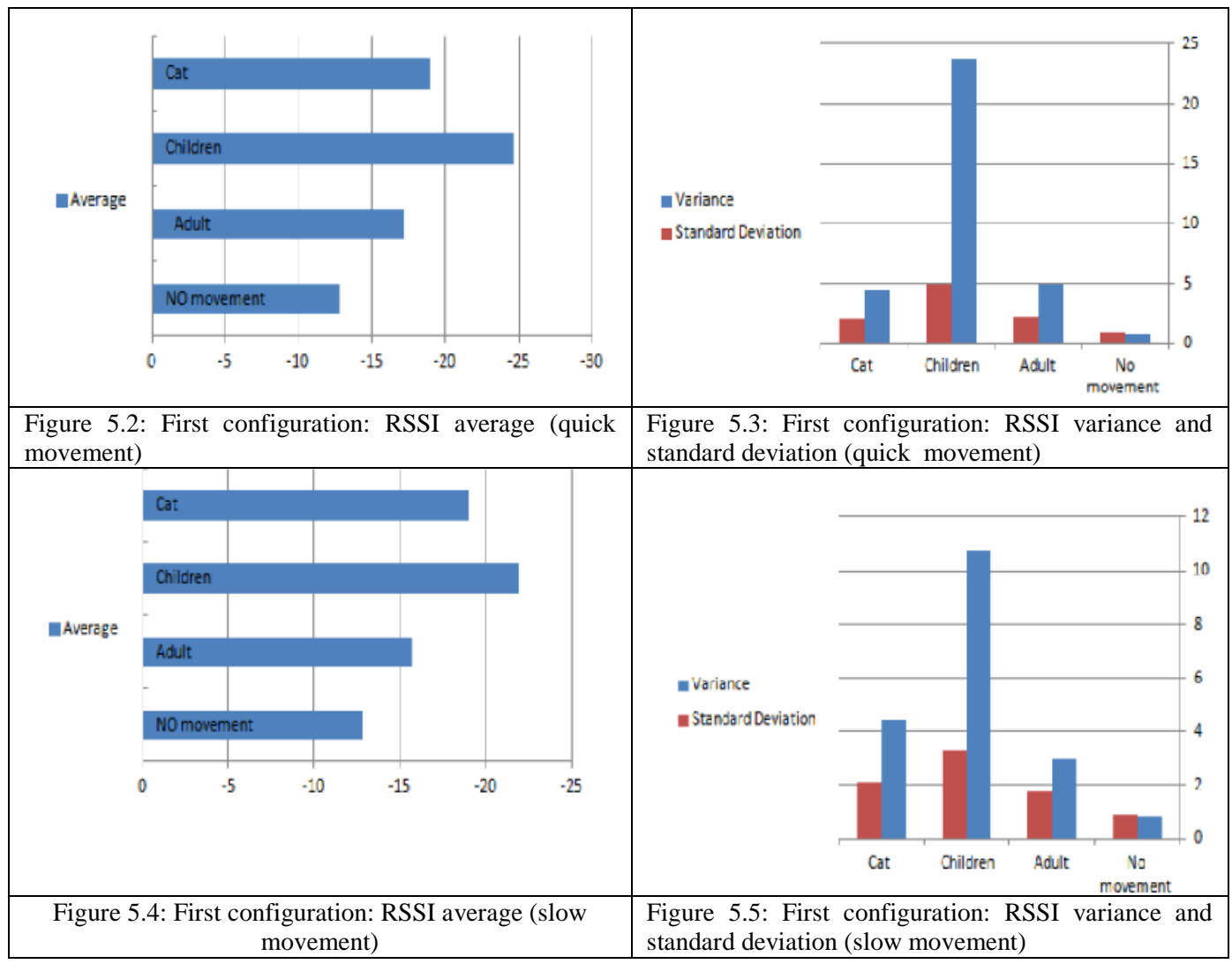

\subsection{Second Configuration}

In this configuration, sensors are away from Base Station two meters and Base-Station rises from the ground 1 meter. The configuration is shown below in Figure 5.6. 
International Journal of Computer Networks \& Communications (IJCNC) Vol.4, No.6, November 2012

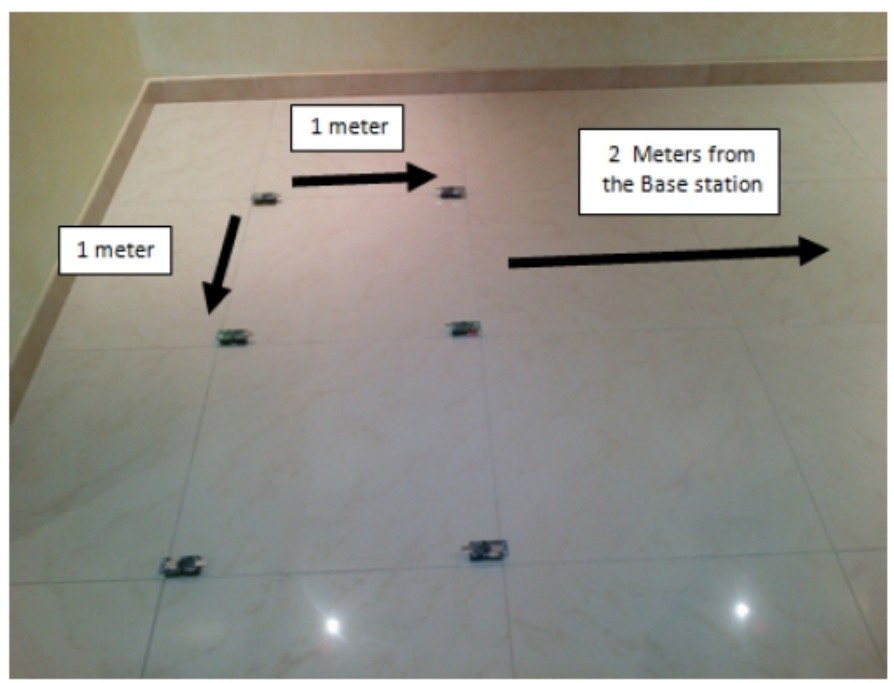

Figure 5.6: Second Sensor Configuration

In this configuration 21 experiences have been done. The Cases are:

1. Adult (Quickly movement): 3 tests.

2. Adult (Slow movement): 3 tests.

3. Children (Quick movement): 3 tests.

4. Children (Slow movement): 3 tests.

5. Cat: 3 tests.

6. Obstacle: 3 tests.

The analysis of these tests is shown below in Figure 5.7, 5.8, 5.9 and 5.10. And Table 5.2 illustrates the RSSI range for each experiment.

Table 5.2: Second configuration testing data

\begin{tabular}{|l||c|c|c|}
\hline Tested Object & Test 1 & Test 2 & Test3 \\
\hline adult (Quickly) & -4 to -27 & -3 to -34 & -4 to -35 \\
\hline adult (Slowly) & -3 to -33 & -2 to -40 & +2 to -45 \\
\hline child (Quickly) & -5 to -30 & -5 to -29 & -5 to -33 \\
\hline child (Slowly) & -6 to -40 & -6 to -32 & -5 to -34 \\
\hline cat & -4 to -25 & -8 to -14 & -5 to -15 \\
\hline obstacle & -8 to -30 & -8 to -22 & -8 to -15 \\
\hline no movement & -8 to -16 & -9 to -17 & -7 to -18 \\
\hline
\end{tabular}

As a result of this several experiments based on average (mean), variance and standard deviation show in Figure 5.7, Figure 5.8, Figure 5.9 and Figure 5.10. It is found that if RSSI $\leq 23$. There is a high probability of intrusion activity at home. Therefore SMS and Email are sent to homeowner if $\operatorname{RSSI} \leq 23$. 
International Journal of Computer Networks \& Communications (IJCNC) Vol.4, No.6, November 2012

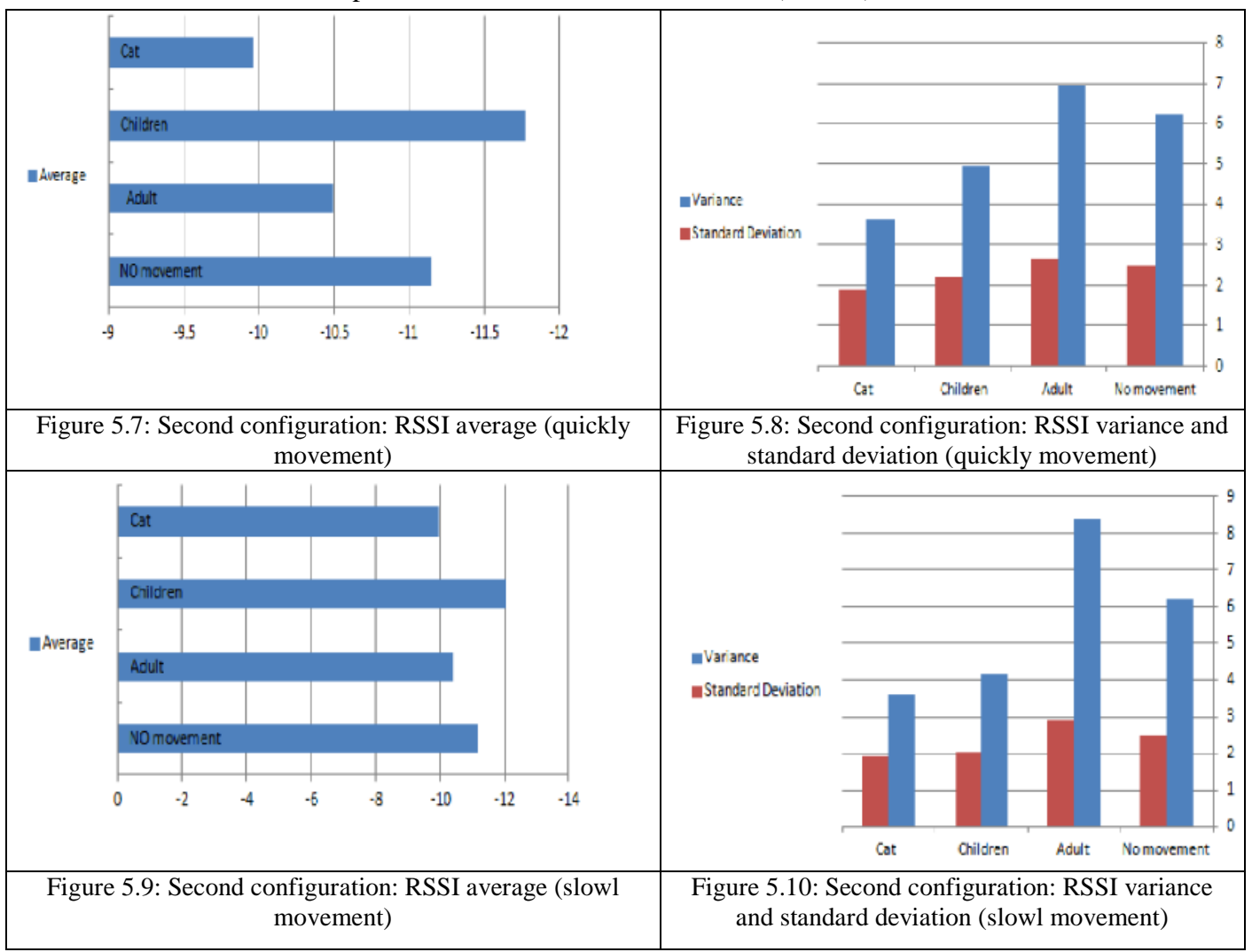

\subsection{Third Configuration}

In this configuration, sensors are away from Base-Station one meter and Base-Station rises from the ground 1 meter. The configuration is shown in Figure 5.11. Table 5.3 illustrates the RSSI range for each experiment.

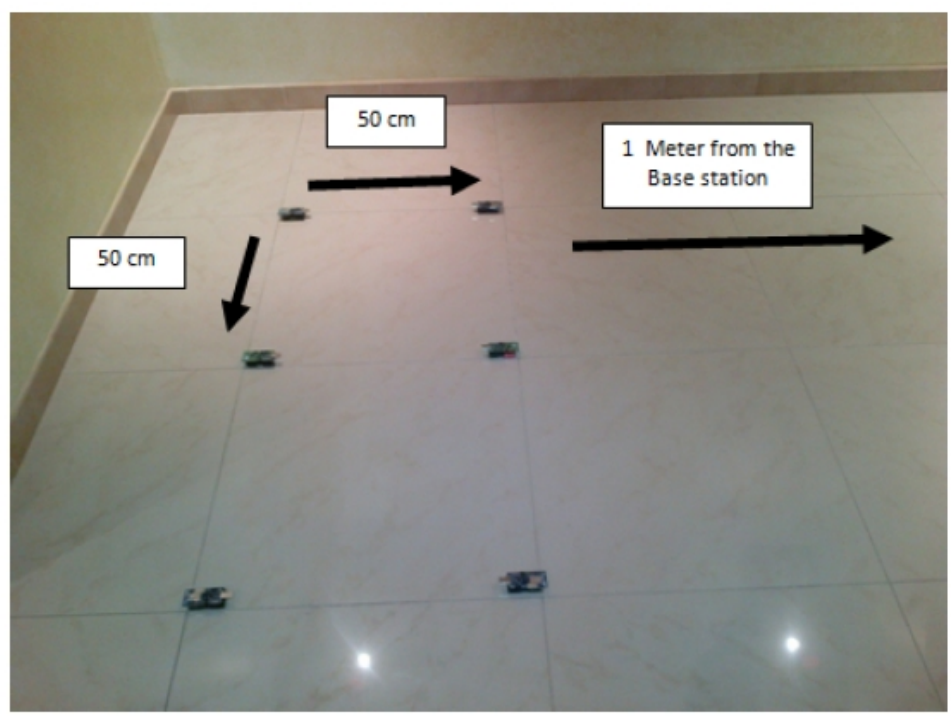

Figure 5.11: Third Sensor Configuration 
International Journal of Computer Networks \& Communications (IJCNC) Vol.4, No.6, November 2012

Table 5.3: Third configuration testing data

\begin{tabular}{|l||c|c|c|}
\hline Tested Object & Test 1 & Test 2 & Test3 \\
\hline adult (Quickly) & 2 to -21 & 2 to -21 & 2 to -21 \\
\hline adult (Slowly) & 3 to -23 & 2 to -23 & 2 to -23 \\
\hline child (Quickly) & 4 to -17 & 4 to -17 & 4 to -25 \\
\hline child (Slowly) & 3 to -17 & 2 to -36 & 3 to -31 \\
\hline cat & 2 to -24 & 2 to -19 & 2 to -16 \\
\hline obstacle & -3 to -23 & -3 to -23 & -3 to -21 \\
\hline no movement & 0 to -9 & 0 to -10 & 0 to -10 \\
\hline
\end{tabular}

As a result of several experiments based on average (mean), variance and standard deviation as shown in Figure 5.12, Figure 5.13, Figure 5.14 and Figure 5.15. It is found that if RSSI $₫$ s, there is a high probability of intrusion activity at home. Therefore SMS and Email are sent to homeowner if RSSI $\leq 5$.

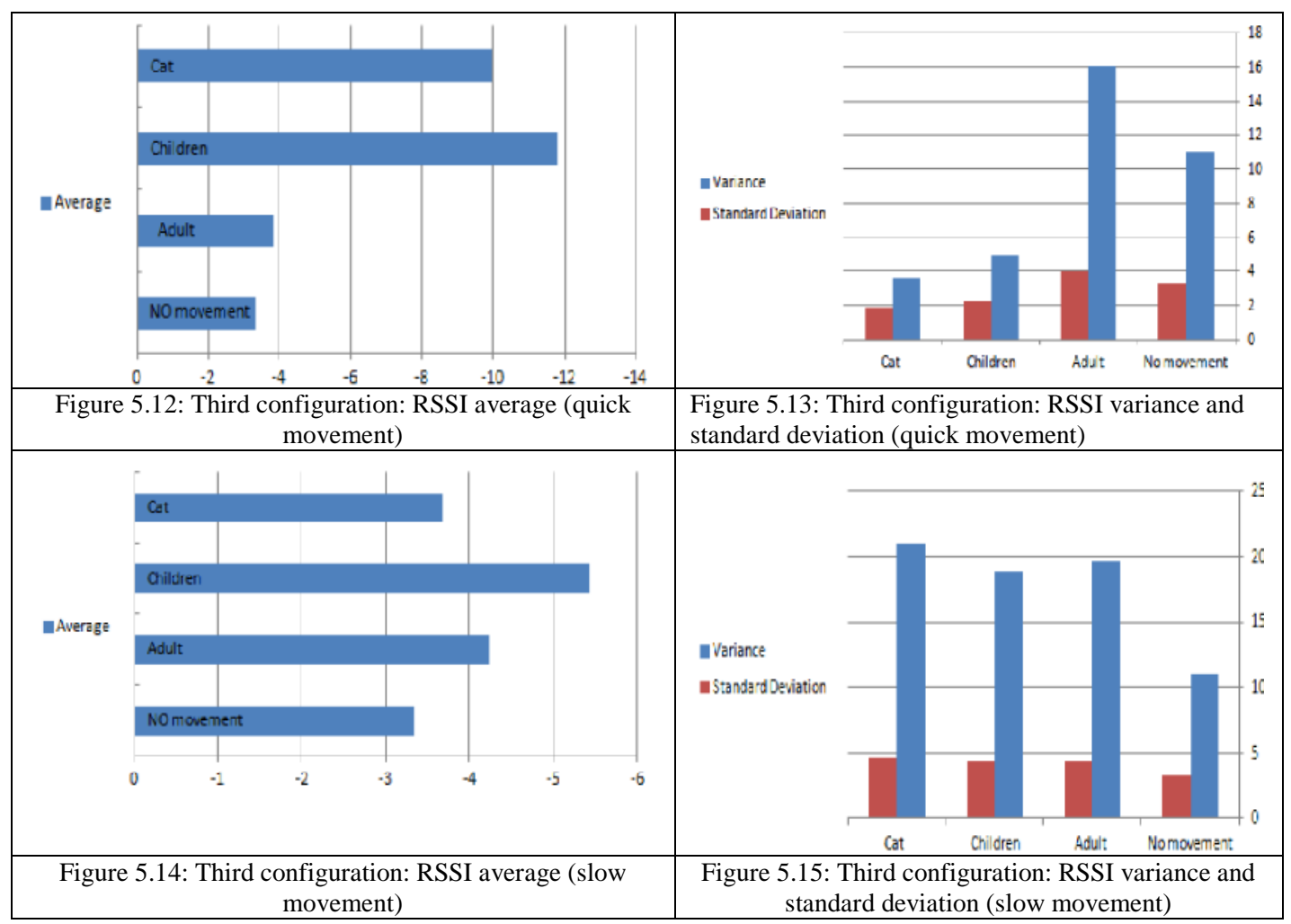

\section{PERFORMANCE ANALYSIS}

In this section we determine the intrusion activity at home by calculating the false rate (positive and negative) based on RSSI values. False positive means that no intrusion activity at home but system sends notification messages. False negative means when there is an intrusion activity at home and the system does not send notification messages. In order to calculate the false rate, the 
International Journal of Computer Networks \& Communications (IJCNC) Vol.4, No.6, November 2012 first configuration as shown in figure 5.1 is considered. Table 5.4 illustrates the experiment result and Table 5.5 illustrates the false negative and false positive result. Figure 5.16 shows false positive and false negative rate.

Table 5.4: Experiment Result

\begin{tabular}{|l|c|c|c|c|c|}
\hline RSSI range & Time & Speed & Temperature & False positive & False negative \\
\hline RSSI $<-19$ & $15 \mathrm{~min}$ & $1 \mathrm{~min}$ & $20 \mathrm{C}$ & $8.7 \%$ & $5 \%$ \\
\hline RSSI $<-34$ & $15 \mathrm{~min}$ & $1 \mathrm{~min}$ & $20 \mathrm{C}$ & $0 \%$ & $100 \%$ \\
\hline RSSI $<-27$ & $15 \mathrm{~min}$ & $1 \mathrm{~min}$ & $20 \mathrm{C}$ & $1 \%$ & $80 \%$ \\
\hline RSSI $<-24$ & $15 \mathrm{~min}$ & $1 \mathrm{~min}$ & $20 \mathrm{C}$ & $1 \%$ & $20 \%$ \\
\hline RSSI $<-23$ & $15 \mathrm{~min}$ & $1 \mathrm{~min}$ & $20 \mathrm{C}$ & $4 \%$ & $20 \%$ \\
\hline
\end{tabular}

Table 5.5: False negative and false positive result

\begin{tabular}{|c||c|c|}
\hline RSSI range & False positive & False negative \\
\hline RSSI $<-19$ & X & $\checkmark$ \\
\hline RSSI $<-34$ & $\checkmark$ & X \\
\hline RSSI $<-27$ & $\checkmark$ & X \\
\hline RSSI $<-24$ & $\checkmark$ & $\checkmark$ \\
\hline RSSI $<-23$ & $\checkmark$ & $\checkmark$ \\
\hline
\end{tabular}

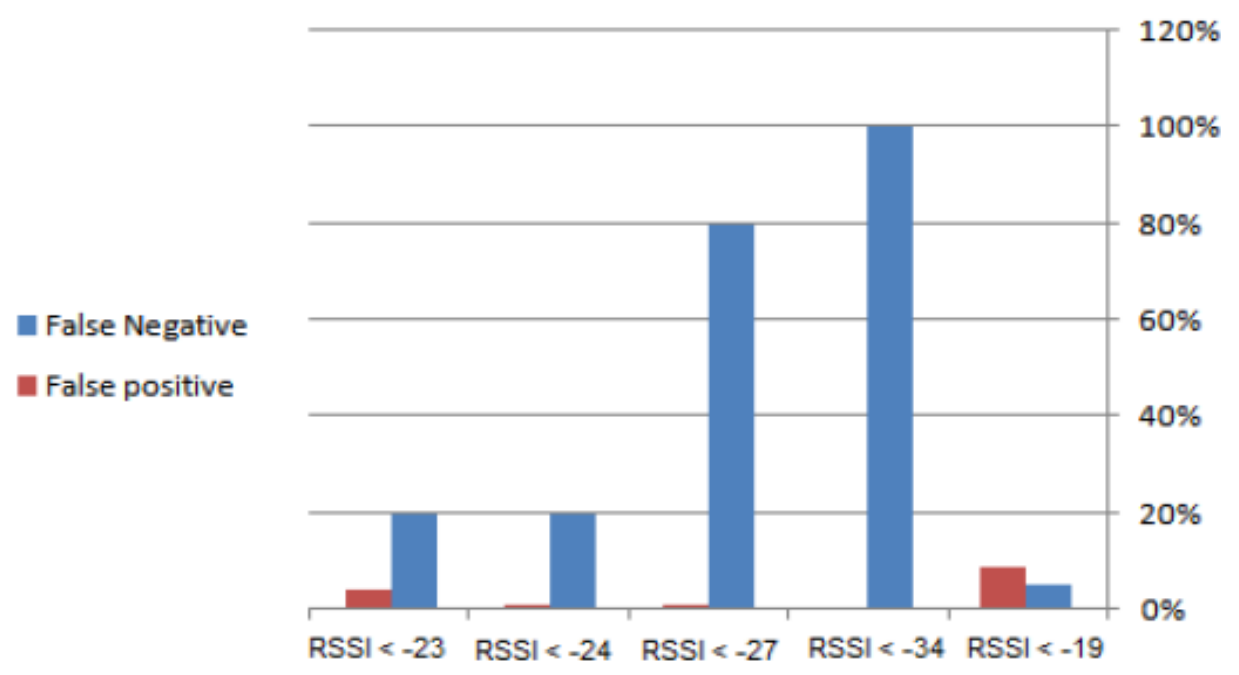

Figure 5.16: False positive and false negative rate

As a result we can say that our proposed system effectiveness depends $60 \%$ on sensor node numbers, sensor nodes quality, experiment place, and the false rate result.

\subsection{Performance Comparisons}

This section presents a comparison between our proposed system and Intelligent Monitoring using Wireless Sensor Networks [14] system. The comparison is made by separately examining each component of both systems in terms of performance. Table 5.6 illustrates the comparisons between both systems. 
International Journal of Computer Networks \& Communications (IJCNC) Vol.4, No.6, November 2012

Table 5.6: Performance Comparison

\begin{tabular}{|l||cc|}
\hline Property & Proposed System & $\begin{array}{c}\text { Intelligent Monitoring } \\
\text { using WSN }\end{array}$ \\
\hline Notifications & $\begin{array}{c}\text { Send notifications by } \\
\text { SMS and l'mail }\end{array}$ & Does not send \\
\hline Functionality & $\begin{array}{c}\text { For intrusion activity } \\
\text { (Children, Adult or cat) }\end{array}$ & $\begin{array}{c}\text { For Localization and } \\
\text { monitoring human } \\
\text { behaviour in a bedroom }\end{array}$ \\
\hline $\begin{array}{l}\text { Configuration } \\
\text { numbers }\end{array}$ & $\begin{array}{c}3 \text { configuration } \\
\text { for monitoring }\end{array}$ & $\begin{array}{c}\text { 1 configuration for } \\
\text { localization and } \\
\text { monitoring }\end{array}$ \\
\hline Tested Object & 3 object types & 1 object type \\
\hline Sensors Number & 6 sensors for monitoring & $\begin{array}{c}4 \text { sensors for localization } \\
\text { and } 9 \text { for monitoring }\end{array}$ \\
\hline Purposc & Home Sccurity Monitoring & $\begin{array}{c}\text { Human bchaviour localization } \\
\text { und monitoring using RSSI }\end{array}$ \\
\hline
\end{tabular}

\section{CONCLUSION}

This paper presents a RHSM system using WSNs. The WSN is a wireless network comprising of distributed independent devices that is used to monitor environmental or physical situations via sensors. The self-sufficient nodes or devices combine with gateway and routers to build a WSN system. We use variations in received signal strength indicator (RSSI) value to find the intrusion activity at home by determining the mobility and have found that accurate intruder detection is possible for at least short distances. Architecture of an application has been presented consisting of Moteivs Tmote Sky motes and a base station. The application sends a piece of alarm as (SMS) an Email message to the homeowner in case of any intrusion activity has been detected at home. So, the people will be able to keep track of their homes' security and protection wherever they are.

\section{References}

[1] E. Coca, V. Popa, G. Buta, "Wireless Sensor Network Nodes Performance Measurements and RSSI Evaluatio,"Design and Technology of Electronics Packages, (SIITME) 2009 15th International Symposium for;pp 105 - 112,17-20 Sept. 2009.

[2] Mainwaring, A., et al., Wireless Sensor Networks for Habitat Monitoring, in ACM International Workshop on Wireless Sensor Networks and Applications (WSNA'02). 2002.

[3] Subhas C. Mukhopadhyay*, Anuroop Gaddam and Gourab S. Gupta, Wireless Sensors for Home Monitoring - A Review, 2008, 1, 32-39.

[4] Wei-Chung Teng, Yu-Chun Pao, Sheng-Luen Chung.(2008). "Design of MyServer: a Residential Server in Smart Home Systems". In: Asia-Paci_c Services Computing Conference, APSCC '08. IEEE, Dec. 2008, pp. 580586.

[5] Kim, S., Pakzad, S., Culler, D., Demmel, J., Fenves, G., Glaser, S., and Turon, M. (2007). "Health Monitoring of Civil Infrastructures Using Wireless Sensor Networks". In :The Proceedings of the 6th International Conference on Information Processing in Sensor Networks (IPSN '07), Cambridge, MA, April 2007, ACM Press, pp. 254-263. 
International Journal of Computer Networks \& Communications (IJCNC) Vol.4, No.6, November 2012

[6] Alan Mainwaring, Joseph Polastre,Robert Szewczyk,David Culler, John Anderson, Wireless Sensor Networks for Habitat Monitoring, WSNA02, September 28, 2002.

[7] Huiping Huang, Shide Xiao, Xiangyin Meng, Ying Xiong,A Remote Home Security System Based on Wireless Sensor Network and GSM Technology, 2010.

[8] Jianjun Chen, Zoltan Safar, John Aa. Srensen and Kare J. Kristofiersen, An RFI based Surveillance System Using Commercial Off-The-Shelf Wireless LAN Component ,.

[9] Zatout, Y., Campo, E., Llibre, J.-F.,"WSN-HM: Energy-efficient Wireless Sensor Network for home monitoring ", pp. 367 372, February 2010.

[10] Dong-Sun Kim, Seung-Yerl Lee, Kwang-Ho Won, Duck-Jin Chung, and Jae-Ho Kim," Time-synchronized Forwarding Protocol for Remote Control of Home Appliances Based on Wireless Sensor Network,", Vol. 53,pp. 1427 1433, January 2008.

[11] A. Gaddam, S. C. Mukhopadhyay, G. Sen Gupta," Integrating a Bed Sensor in a Smart Home Monitoring System," , pp. 518 - 521 , June 2008.

[12] Yanjun Li; Zhi Wang; Yeqiong Song; , "Wireless Sensor Network Design for Wildfire Monitoring," Intelligent Control and Automation, 2006. WCICA 2006. The Sixth World Congress on , vol.1, no., pp.109-113.

[13] Yu-Tso Chen, Chi-Lu Yang, Yeim-Kuan Chang, Chih-Ping. " A RSSI-based Algorithm for Indoor Localization Using ZigBee in Wireless Sensor Network".

[14] Erdogan, Senol Zafer, Hussain, Sajid, Park, Jong Hyuk, Intelligent Monitoring Using Wireless Sensor Networks, The 3rd IFIP International Symposium on Network Centric Ubiquitous System (NCUS 2007) LNCS, Springer-Verlag, Taipei, Taiwan, December 17-20, 2007. 